Der Bundesrat hatte per 1. August 2007 die Verordnung zum KVG um einen Art. 59c mit Regeln für die Tarifgenehmigung ergänzt. Er tat dies, weil neu das Bundesverwaltungsgericht für den Entscheid über Tarifbeschwerden zuständig wurde. Die FMH hatte von Anfang an den Eindruck, die neue Bestimmung zeuge von einem eigenartigen Verständnis von Bundesverfassung, KVG und Betriebswirtschaft. Das BAG hat sich im Zusammenhang mit der Revision der Analyenliste explizit auf diesen Artikel abgestützt und zudem die Auffassung vertreten, «Kosten decken» bedeute, das Präsenzlabor des Arztes dürfe keinen Deckungsbeitrag für beanspruchte Leistungen aus dem allgemeinen Teil der Arztpraxis leisten. Art. 59c KVV wird auch bei Festsetzungsverfahren im LeiKoV-Bereich und bei Neutarifierungen im Bereich des TARMED immer wieder zitiert bzw. als Grundlage verwendet. Deshalb hat die FMH bei Prof. Regula Kägi-Diener ein Rechtsgutachten zu diesen für uns äusserst wichtigen und brisanten Fragen in Auftrag gegeben. Dieses kommt in klaren Worten zu eindeutigen Schlüssen ...

\title{
«Eine Vollziehungsverordnung [darf] nicht über den Rahmen hinausgehen, den das Gesetz absteckt»*
}

\section{Hanspeter Kuhn}

Fürsprecher, stv. Generalsekretär $\mathrm{FMH}$

\footnotetext{
Zusammenfassung des Gutachtens von Prof. Regula Kägi-Diener zur Verfassungs- und Gesetzmässigkeit von Art. 59c KVV.
}

Korrespondenz:

Rechtsdienst FMH

Postfach 170

CH-3000 Bern 15

Tel. 0313591111

Fax 0313591112

lex@fmh.ch
Die Gutachterin sollte untersuchen, ob Art. 59c KVV mit dem KVG und der Verfassung vereinbar ist bzw. wie er gesetzes- und verfassungskonform gelesen werden muss.

Art. 59c KVV (Tarifgestaltung) lautet wie folgt:

1 Die Genehmigungsbehörde im Sinne von Artikel 46 Absatz 4 des Gesetzes prüft, ob der Tarifvertrag namentlich folgenden Grundsätzen entspricht:

a. Der Tarif darf höchstens die transparent ausgewiesenen Kosten der Leistung decken.

b. Der Tarif darf höchstens die für eine effiziente Leistungserbringung erforderlichen Kosten decken.

c. Ein Wechsel des Tarifmodells darf keine Mehrkosten verursachen.

2 Die Vertragsparteien müssen die Tarife regelmässig überprüfen und anpassen, wenn die Erfüllung der Grundsätze nach Absatz 1 Buchstaben $\mathrm{a}$ und $\mathrm{b}$ nicht mehr gewährleistet ist. Die zuständigen Behörden sind über die Resultate der Überprüfungen zu informieren.

3 Die zuständige Behörde wendet die Absätze 1 und 2 bei Tariffestsetzungen nach den Artikeln 43 Absatz 5, 47 oder 48 des Gesetzes sinngemäss an.

Hier sind die wichtigsten Auszüge aus dem Gutachten (Zwischentitel vom Autor).

\section{Eine Ausführungsverordnung darf keine Rechte beschränken}

«Das Bundesgericht hat in BGE 103 IV 192, E. 2a, 194 die Grenzen für die Vollzugskompetenz eng umschrieben. Danach darf eine Vollziehungsverordnung nicht über den Rahmen hinausgehen, den das Gesetz absteckt; sie hat bestimmungsgemäss keine andere Funktion als gewisse Bestimmungen des Gesetzes zu präzisieren, gegebenenfalls echte Lücken zu füllen und, soweit notwendig, das anwendbare Verfahren festzulegen. Entsprechend darf sie keine neuen Vorschriften enthalten, welche den Anwendungsbereich des Gesetzes ausdehnen und Rechte der Adressatinnen und Adressaten beschränken oder ihnen Verpflichtungen auferlegen, selbst wenn diese Regeln dem gesetzgeberischen Ziel des Gesetzes entsprechen würden.»

\section{Das KVG will keine Staatsmedizin einführen}

«Beim Inkrafttreten des geltenden KVG im Jahre 1996 wurde eine stringente Regulierung für Krankenkassen und Tarife eingeführt, auch das damals totalrevidierte KVG hob aber den liberalen Gedanken der schweizerischen Rechtsordnung nicht völlig aus den Angeln. [...] Selbst bei den Tarifen betonte der Bundesrat damals: «Es steht ... das Prinzip der Vertragsfreiheit im Vordergrund (Botschaft KVG S. 179 oben, Separatdruck S. 87 oben). Es war deshalb selbst dem Bundesrat klar, dass grundsätzlich die Wirtschaftsfreiheit Geltung haben sollte und dies war eines der erklärten Elemente des revidierten Gesetzes.

Es kommt noch folgendes hinzu: Trotz mannigfaltiger Einschränkungen für die Leistungserbringer durch das KVG müssen ausserhalb der öffentlichen Spitäler Ärztinnen und Ärzte, aber auch andere Leistungserbringer, unter privatwirtschaftlichen Bedingungen ihr Einkommen 
als Unternehmerinnen und Unternehmer generieren. Dieses Modell liegt dem KVG zugrunde, denn es war nie beabsichtigt, eine Staatsmedizin einzuführen. [...] Die Wirtschaftsfreiheit (Art. 27 $B V)$ ist deshalb zu beachten, soweit das KVG sie nicht ausdrücklich beschränkt.»

\section{Das «höchstens» irritiert - nicht- kostendeckende Tarife wären system- widrig und unverständlich}

«Liest man Art. 59c Abs. 1 KVV unvoreingenommen, irritiert in den lit. a und b sodann der Begriff 〈höchstens`: Der Tarif dürfe, um genehmigt zu werden, höchstens〉 die transparent ausgewiesenen und für eine effiziente Leistungserbringung erforderlichen Kosten decken. Dies soll bei Tariffestsetzungen sinngemäss gelten (Art. 59c Abs. 3 KVV). Das liest sich - jedenfalls auf den ersten Blick - so, als könnte der Tarif auch weniger als die ausgewiesenen und unter Effizienzaspekten erforderlichen Kosten der Leistung decken. Das würde gleichzeitig bedeuten, dass den Leistungserbringern mit jeder Leistung Kosten entstehen, die sie nicht überwälzen können. Sie haben nämlich einen Tarifschutz der Patientinnen und Patienten zu respektieren (Art. 44 KVG) und sind deshalb an den Tarif gebunden. Aufgrund des (deutschen) Wortlautes würden bei diesem Verständnis die technische und/oder die ärztliche Leistung nicht vollumfänglich vergütet und unwirtschaftlich werden. Dies wäre mit Rücksicht darauf, dass Praxisärzte (und andere private Leistungsträger) nicht subventioniert werden, in einem privatwirtschaftlichen - wenngleich regulierten - System nicht nur ungewöhnlich, sondern systemwidrig und unverständlich. [...] Mit Art. 43 Abs. 4 KVG hat der Gesetzgeber nun aber vorgeschrieben, dass die Tarife nach KVG primär betriebswirtschaftlich $\mathrm{zu}$ bemessen sind (gleichzeitig müssen sie sachgerecht ausgestaltet sein).» [1]

\section{Kosten der Modellpraxis müssen massgebend sein}

«Bei der Bemessung der betriebswirtschaftlichen Kosten gemäss Art. 43 Abs. 4 KVG wird nicht auf hypothetische Kosten abgestellt, sondern auf repräsentative Kostendaten, die auf Erhebungen über die effektiven Kosten in den ärztlichen Praxen beruhen [2] (sog. Modellpraxis). Die so ermittelten Durchschnittskosten sollen eine aufwand- und verursachergerechte Leistungsentschädigung bei optimaler Auslastung ergeben [3].

[...] eine Genehmigungs- (bzw. Festsetzungs-)Behörde darf nur dann unter die betriebswirtschaftlich ermittelten Kosten gehen, wenn sie gute Gründe hat, dass diese zu hoch sind, um als «wirtschaftlich〉 zu gelten. Zumindest muss sie nachweisen, dass sich (auch) die abweichenden Kosten, die sie dem Tarif zugrunde legen will, in vertretbarer Weise betriebswirtschaftlich begründen lassen und für die Leistungserbringer zumutbar sind. Und selbstverständlich muss sie den betroffenen Kreisen Gelegenheit geben, zu solchen Gründen Stellung zu nehmen, und ihre Einwände in Erwägung ziehen.»

\section{Bundesgericht verbietet «Frondienste» für Berufsstand}

«Das Bundesgericht hat in einem Entscheid betreffend das Einkommen von Anwälten und Anwältinnen in der unentgeltlichen Rechtspflege ausgeführt, es sei einem Berufsstand nicht zuzumuten «Frondienste〉 zu leisten, es erscheine «stossend, wenn ihnen für diesen Teil der Tätigkeit bloss die eigenen Aufwendungen abgegolten werden. Und das Bundesgericht führte weiter aus: ¿Es ist mit dem Willkürverbot und indirekt auch mit Art. 27 BV nicht mehr vereinbar, als Untergrenze für eine angemessene Entschädigung lediglich die Deckung der Selbstkosten vorzuschreiben.> [4] Diese Ausführungen erfolgten wohlgemerkt zu einer Tätigkeit, welche regelmässig lediglich einen kleineren Anteil an der gesamten Berufstätigkeit beschlägt. Für Ärzte dagegen wird die gesamte Berufstätigkeit über die Tarife geregelt. Wenngleich hier die Frage des Referenzeinkommens für die Kostenberechnung nicht weiter vertieft werden kann, muss doch, um dem Grundsatz der Wirtschaftsfreiheit zu genügen, bei der Kostenberechnung auf vergleichbare Einkommen mit vergleichbaren Grundvoraussetzungen abgestellt werden.»

\section{Kostenneutralität bei Wechsel des Tarifmodells: fehlende gesetzliche Ermächtigung}

«Art. 59c Abs. 1 lit. c KVV legt fest, dass ein Wechsel des Tarifmodells keine Mehrkosten verursachen dürfe. Es wird hier die sogenannte Kostenneutralität für Tarifwechsel statuiert. Dabei ist zu bemerken, dass diese Kostenneutralität jedenfalls nach dem Wortlaut einseitig gelten soll, das heisst nur keine Mehrkosten entstehen dürfen. Ob Minderkosten zulässig sind, lässt die Bestimmung offen (eine «echte> Neutralität würde diese nicht zulassen). [...]

Die Beschränkung der Vertragsautonomie ist gleichzeitig eine Beschränkung der Wirtschaftsfreiheit und darf deshalb nicht in einer Verordnung statuiert werden, wenn - wie hier - das Gesetz den Bundesrat nicht dazu ermächtigt.» 
Fazit 1: Wer Verfassung und Gesetz respektiert, liest Art. 59c Abs. 1 lit. a und b KVV wie folgt

«Die Genehmigungsbehörde im Sinne von Artikel 46 Absatz 4 des Gesetzes beachtet bei der Genehmigung die Vertragsautonomie der Tarifpartner und prüft, ob der Tarifvertrag namentlich folgenden Grundsätzen entspricht:

(a. und b.) Der Tarif hat den transparent ausgewiesenen Kosten der effizient erbrachten Leistung zu entsprechen. Zu diesen Kosten gehört ein angemessenes Unternehmereinkommen. Er kann unter die transparent ausgewiesenen Kosten der Leistung gehen, wenn diese Kosten nicht dem Wirtschaftlichkeitsgebot entsprechen. Die Abweichung muss betriebswirtschaftlich begründbar, verhältnismässig und für die Leistungserbringer zumutbar sein.»

Fazit 2: Art. 59c Abs. 1 lit. c KVV (Kostenneutralität bei Tarifmodellwechsel): Kompetenzen überschritten

«[Dieser Absatz] dürfte [...] die Schranken für die allgemeine Vollzugskompetenz des Bundesrates in dieser Sache sprengen. Er geht über die Anfor- derungen hinaus, die das KVG selber an einen Tarifwechsel stellt.»

\section{Literatur}

1 Zur «sachgerechten Struktur» führte die Expertenkommission Schoch, Bericht vom 2. November 1990 (Sonderdruck S. 60), seinerzeit aus: «Durch die Vereinheitlichung der Tarifstrukturen sollen vor allem folgende Ziele erreicht werden: eine angemessene Wertrelation unter den Leistungen aufgrund betriebswirtschaftlicher Bemess-ungskriterien (Art. 31 Abs. 1).»

2 Siehe dazu Gebhard Eugster, Krankenversicherung, Rz. 884 und ff., in: Ulrich Meyer (Hrsg.), Soziale Sicherheit, SBVR, 2. Aufl., Basel/Genf/München 2007.

3 Siehe dazu: Beatrice Gross Hawk, Selbständige nicht ärztliche Medizinalpersonen in der freien Praxis, Schulthess Zürich 2008, Rz. 107.

4 So BGE 2P.17/2004 und 2P.325/2003 vom 6.6.2006; siehe auch BGE $131 \mathrm{~V} 153$. 